\title{
A Survey on QoS Behavior in MPLS Networks
}

\author{
Shruti Thukral ${ }^{1}$, Banita Chadha ${ }^{2}$ \\ M.Tech Scholar, CSE Department, IEC College of Engg \& Technology, Greater Noida, India ${ }^{1}$ \\ Assistant Professor, CSE Department, IEC College of Engg \& Technology, Greater Noida, India ${ }^{2}$
}

\begin{abstract}
Quality of Service (QoS) in Real Time Applications such as video, voice, online gaming is becoming the major concern. In Today's Scenario Voice over Internet Protocol (VoIP) is an emerging service because of the benefits of this service. The most advantageous benefit which attracts many people using this service is the low cost. QoS is an important factor for the voice traffic. Internet Protocol (IP) network implements QoS in two ways i.e. Integrated Services (IntServ) and Differentiated Services (DiffServ) but still VoIP suffers from delay, voice echo, packets loss or Intermittent silent pauses. Multi Protocol Label Switching (MPLS) is a framework specified by IETF when used with IP increases its QoS. MPLS is used as an extension to IP. In this paper we define the MPLS QoS management and how it supports the IP QoS architecture.
\end{abstract}

Keywords: Internet Protocol (IP), Multi Protocol Label Switching (MPLS), Quality of Service (QoS), IntServ, DiffServ, Traffic Engineering (TE)

\section{INTRODUCTION}

Networks have become a rudimentary. They have become an important component of life. More and more people are switching towards the Internet. Internet is constantly growing among service providers and customers. As the usage of Internet increases and become more popular, there is more Traffic in the network. The Internet Protocol (IP) is the Dominant protocol for sending the data from source to the destination. Some existing services require high level of Quality of Service and impose great demands on the network such as real time applications such which are very sensitive to delay and jitter and require highcapacity bandwidth. In the last few years the users of Internet Telephony services have increased tremendously. Voice over Internet Protocol (VoIP) is the largely supported service used for the voice application because of its benefits such as it is cost effective. IP uses IntServ and DiffServ architecture to provide QoS. Most popular and in use is the DiffServ architecture of IP. A lot of people and organizations around the world are devoting their attention to the service quality. The popularity of VoIP has grabbed the interest of many researchers because of its low infrastructure cost. Quality of Service in VoIP is the key interest of researchers as it is sensitive to delay, packet loss, voice echoes, or intermittent silent pauses. Various network protocols and architectures supporting QoS assurance are available now and are still being developed. Multi protocol label switching (MPLS) is a new framework emerging in the society to extend the QoS of IP. It is the extension of IP not the replacement. It works to solve the shortcoming of IP.

MPLS is a technology used for speeding up the traffic flow of the network. MPLS uses Label Switching technique for forwarding the packets in the MPLS domain thus speeding up the forwarding mechanism as Labels are short unique identifiers. IP forwards the packets by looking up at the destination address in the packet which is time consuming task in comparison to label lookup performed by MPLS. MPLS makes it easy to create "virtual links" between distant nodes. As the name suggests. It has the capability of working with different protocols and can encapsulate packets of various network protocols. In MPLS, Packet-forwarding decisions are made solely on the contents of this label, there is need to examine the whole packet. This allows one to create endto-end circuits across any type of transport medium, using any protocol. Since MPLS by itself cannot provide service differentiation, combination of DiffServ with MPLS architectures seems to be a useful solution to provide QoS to multimedia traffic while effectively using network resources [3]. The result of this integration is the DiffServaware Traffic Engineering (DS-TE) [3].

This paper mainly focuses on the QoS and its functionality and how MPLS deploy QoS. The first section describes the meaning of QoS and its different models and also explains which model is better to support QoS. The section also explains the different QoS functions. The second section describes the deployment of QoS in IP networks. The third section briefly explains the terminology and working of MPLS networks. The fourth section explains the QoS deployment in MPLS networks. The fifth and the last section conclude the whole survey.

\section{Quality Of Service (QOS)}

QoS has become popular the past few years. It is defined as set of techniques to classify and manage network resources with the help of which certain level of packet loss, jitter, delay etc., can be guaranteed. It is a means to prioritize important traffic over less important traffic and make sure it is delivered. During the past several years, various mechanisms have developed for providing QoS for communication networks as shown in Fig. 1 [1]. The Internet Engineering Task Force (IETF) has proposed many service models and mechanisms to meet the demand for QoS. The most popular models are: the best effort service model, IntServ Service model, DiffServ Service model. The Best Effort Service model is the default model used by IP which does not guarantee QoS. 
- $\quad$ Best Effort Service Model: This is a single traffic conditioning is needed. The configuration of border service model. In this model, an application does not give router is carried out by taking a traffic profile with the help any prior information to the network or take permission of SLA. Packet forwarding is carried out by core routers from the network before sending the data. It does not examining DSCP and mapping with PHB. As standardized provide any guarantee that the data is delivered. It by the IETF, there are two kinds of PHB's, Expedited provides no QoS.

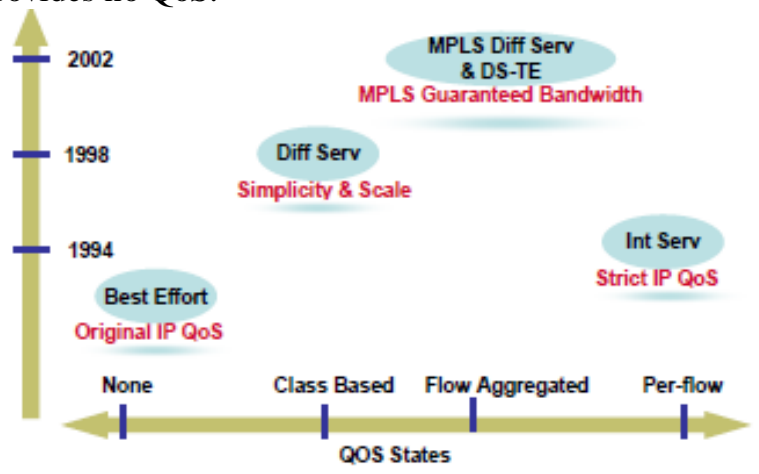

Fig.1. QoS Model

- All IntServ (Integrated Service Model): Integrated service is a multiple service model that can accommodate multiple QoS requirements. In this model, first the request is sent to the network before sending the data. An application request services from the network, after the getting the confirmation from the network the application sends the data. It provides hard QoS. The main problem with the IntServ architecture is the scalability. In the control plane, per-flow information is kept. In the data forwarding plane, per-flow classification, per-flow buffer management and per-flow scheduling are required, which places a huge storage and processing overhead on the routers.

- DiffServ (Differentiated Service Model): Differentiated service is a multiple service model that can satisfy differing QoS requirements. An application using differentiated service does not explicitly signal the router before sending data as in the integrated service model. It overcomes the underlying problem of scalability in IntServ. It provides soft QoS. In DiffServ the classes are divided into different service classes which are treated differently. There is a phenomenon of Behavioural aggregate (BA) in which the group of flows is aggregated which is supposed to make the DiffServ scalable [8]. This phenomenon of BA is carried out by using the different routers with different resources; the functionalities of core routers and the border router are separated. Core routers cannot interchange packet with other domain since they have only access to the internal paths or connections. But if the packet has to be interchanged with the other domains then the border router comes into play. The packets of different BA's are given unique treatment by the routers and this is termed as Per Hop Behaviour (PHB). Differentiated Service Code Point (DSCP) marked in the Differentiated Service (DS) field is used to identify and classify the packets in the DiffServ. Keeping records of per flow information and the traffic conditioning is done by the Border routers. There is profile of certain agreement made for the incoming and outgoing traffic which shouldn't be altered. Hence, for taking care of this and not letting the traffics to get off from the boundary,
Forwarding (EF) and Assured Forwarding (AF) [13].

There are three notions of QoS defined in [14] — intrinsic, perceived, and assessed. Intrinsic QoS pertains to service features stemming from technical aspects. Thus, intrinsic quality is determined by a transport network design and provisioning of network access, terminations, and connections [3]. Intrinsic QoS is evaluated by the comparison of measured and expected performance characteristics. User perception of the service does not influence the intrinsic QoS rating. Perceived QoS reflects the customer's experience of using a particular service. It is influenced by the customer's expectations compared to observed service performance. The assessed QoS starts to be seen when the customer decides whether to continue using the service or not [14]. This decision depends on the perceived quality, service price, and responses of the provider to submitted complaints and problems.

\section{A. QoS Functions}

There are basically two main functions that are:

- Traffic Classification: In this process of traffic classification packets are selected by the classifier. This selection is done by the combination of DSCP value in the IP header. Multi-Field (MF) and the Behavior Aggregate (BA) are the classifiers responsible for this process. Based on the combination field of the IP header, MF selects the packet. In case of $\mathrm{BA}$, selection of packet is done depending on its DSCP value.

- $\quad$ Traffic Conditioning: Another important step is the traffic conditioning, which performs marking, policing, shaping, and metering for confirming the traffic entry to the DiffServ domain. This whole process should be completed satisfying the rules mentioned in the Traffic Conditioning Agreement (TCA) and Service Level Agreement (SLA). Let's understand those functions as described in [12].

Policing: Policing is the process in which the packets are discarded within the traffic stream so as to comply with the rules mentioned in SLA.

Shaping: Shaping is the process in which the packets are delayed so as to comply with the rules mentioned in SLA.

Marking: Marking is the process in which the DSCP value is set in accordance with the set of defined rules like remarking, pre-marking.

$>\quad$ Metering: Metering is the process in which the classifier selects the traffic stream and the temporal properties like rate of those selected traffic stream is measured.

\section{III.QOS IN IP NETWORKS}

Earlier in IP networks IntServ QoS of services is used. In IP header 8 bit Type of Service (ToS) field is defined in which 3 precedence bits were reserved for QoS. Fig.2. explains the IP ToS octet 


\begin{tabular}{|l|l|l|l|l|l|}
\hline Precedence & $D$ & $T$ & $R$ & $C$ & 0 \\
\hline
\end{tabular}

Fig.2. IP Type of Service (ToS) Octet

Where, $\mathrm{D}=$ Delay, $\mathrm{T}=$ Throughput, $\mathrm{R}=$ Reliability, $\mathrm{C}=$ Cost.

The drawback of the precedence bits is that only 3 exist, which means we can have only eight levels of service.

So the DiffServ QoS model comes in play. The DiffServ model is based on redefining the meaning of the 8-bit ToS field in the IP header. The original ToS definition was not widely implemented, and now the field is split into the 6bit DiffServ Code Point (DSCP) value and the 2-bit Explicit Congestion Notification (ECN) part.

Fig.3. describes the DSCP specification

Diffserv Code Point (DSCP)

Fig.3. DiffServ Field Specification

\section{IV.MPLS NETWORKS}

Multiprotocol Label Switching (MPLS) provides high performance packet control and forwarding mechanism for routing the packets in the data networks [2]. It has evolved into an important technology for efficiently operating and managing IP networks because of its superior capabilities in providing traffic engineering (TE) and virtual private network (VPN) services [9]. It is not a replacement for the IP but an extension for IP architecture with including new functionalities and applications. Fig.4.shows architecture of MPLS network.

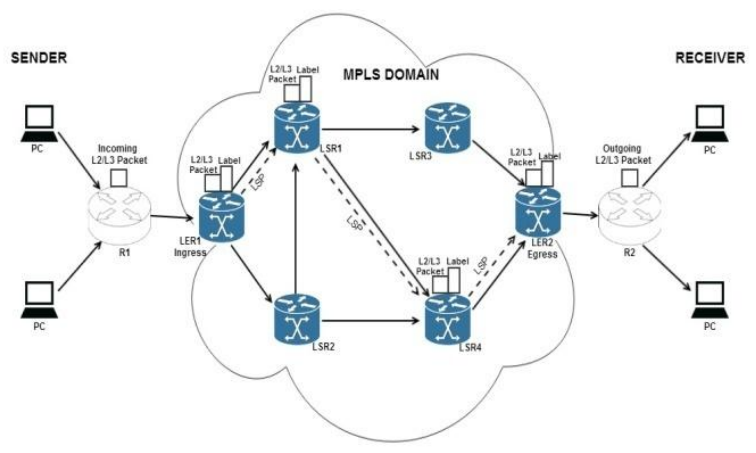

Fig.4. MPLS Networks

Some basic terminologies of MPLS are described below: - Label Switch Router (LSR): LSR is a MPLS node that is capable of forwarding layer 3 packets. This MPLS node or router can operate at the core of the network or at the boundary of the network. If the LSR is at the core of the network then it is used to route the packets by looking up at the label and swaps the label before it is sent to the output port of the node.

- $\quad$ Label Edge Router (LER): If the Label Switch Router (LSR) works at the boundary of the network then it is termed as the Label Edge Router (LER). According to its functionality the LER can be categorized as Ingress
LER and Egress LER. The Ingress router is pushes the Label on the incoming packet and the Egress router pops the label from the packet and delivers it to the destination.

- Label Switched Path (LSP): The LSP is the path formed between the two Label Edge Routers and is used to forward the label packets through this path. This path is established by the signalling protocols in the MPLS domain.

\section{A. Traffic Engineering (MPLS-TE)}

With the standardization of MPLS by IETF, traffic engineering gained its popularity due to the supportive features of the MPLS for traffic engineering far more than the conventional IP networks. The main building blocks of the MPLS Traffic Engineering Model are Path Management, Traffic Assignment, Network State Information Dissemination and Network Management [8]. MPLS Traffic Engineering helps to distribute the flow of traffic evenly on all the links thus avoiding congestion. It attempts to correct the inefficiencies of typical datagram routing protocols. Reengineering a conventional datagram network and moving all data flow across a link to an alternate path can be both expensive and inefficient [8]. A MPLS traffic engineered tunnel is more flexible because when congestion occurs in the network a more desirable route becomes available. The goal of TE is to increase throughput across a network while concurrently decreasing the congestion.

Path Management here means a mechanism by which MPLS network manages the packet forwarding, which includes choosing the right path for the specific packet, maintaining the existing path and finding new paths if some links are added. The path selection is mostly dependent upon the resource attributes, with which the resource and the packets can be categorized and dealt accordingly. The other attribute Traffic Assignment is related to the assignment of traffic to the established tunnel by path management.

The concept of the MPLS Traffic Engineering is explained using the fig 5. this figure shows two paths to travel from Pune to Jaipur. The one path is the direct path from Pune to Jaipur and the other path is Pune-Mumbai- Delhi and then Jaipur.

If we assume all the links have the same cost then traditional IP routing which uses algorithm such as Open Source Shortest Path (OSPF) then the packets are always transferred through the direct link. This makes the direct link very congested and the packets start dropping.

So this link will be over utilized while some links are underutilized. If we use MPLS -TE, a Traffic Engineering tunnel can be established between Pune and Jaipur. It is called a tunnel because the path taken by the traffic is predetermined at the Pune router and not by a hop-by-hop routing decision. 


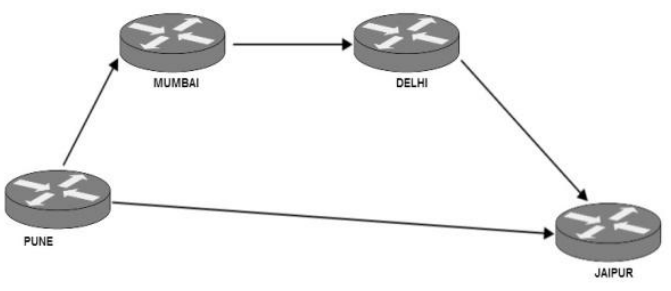

Fig.5. MPLS- TE

\section{QOS IN MPLS NETWORKS}

MPLS does not define new QoS architecture. It uses Differentiated Services (DiffServ) architecture defined for IP QoS (RFC 2475). MPLS DiffServ is defined in RFC3270. In 20 bit MPLS Header the 3 bit EXP field is responsible for handling QoS.

\begin{tabular}{|c|c|c|c|}
\hline Label & EXP & S & TTL \\
$(20$ bits $)$ & $(3$ bits $)$ & $(1$ bit $)$ & $(8$ bits $)$ \\
\hline
\end{tabular}

Fig.6. Example of an image with acceptable resolution

\section{A. DiffServ with MPLS}

Combining the DiffServ-based classification and PHBs with MPLS-based TE leads to true QoS in packet backbones as DiffServ provides a QoS treatment to traffic aggregates. It is a scalable and operationally simple solution as it does not require per- flow signaling and state. However, it cannot guarantee QoS, because it does not influence a packet path, and therefore, during a congestion or failure, even high-priority packets do not get guaranteed bandwidth. MPLS, on the other hand, can force packets into specific paths and - in combination with constraint-based routing - can guarantee bandwidth for FECs. But in its basic form MPLS does not specify classbased differentiated treatment of flows.

MPLS Scalability comes from the aggregation of the traffic on the LER and the processing this traffic in the core network.

When compared to IP DiffServ the Functional Components i.e. TCA and PHB and where they are used remains the same. Buffer management and packet scheduling mechanisms used to implement PHB also remains unchanged.

The main difference between the IP DiffServ and MPLS DiffServ is that Prec/DSCP field is not directly visible to MPLS Label Switch Routers. They forward based on MPLS Header and EXP field. Information on DiffServ must be made visible to LSR in MPLS Header using EXP field / Label.

In a DiffServ domain, all IP packets crossing a link and requiring the same DiffServ behavior are said to constitute a Behavior Aggregate (BA). At the ingress node of the DiffServ domain packets are classified and marked with a DiffServ Code Point (DSCP) which corresponds to their BA. At each transit node, the DSCP is used to select the Per Hop Behavior (PHB) that determines the scheduling treatment and, in some cases, drop probability for each packet. RFC 3270 [9] specifies a solution for supporting the DiffServ BAs whose corresponding PHBs are currently defined over an MPLS network [2], [4]. This solution also offers flexibility for easy support of PHBs that may be defined in the future. In addition, two types of Label Switched Paths (LSPs) have been defined; E-LSP and L-LSP. An E-LSP can carry up to 8 PHBs. The EXP value of an MPLS label (used for packet classification and marking) identifies PHB. Label value is not used for QoS treatment (classification, queuing, dropping, and marking). An L-LSP carries packets belonging to a single PHB Scheduling Class (PSC) identified by the label value. A PSC consists of one or more PHBs where PHBs within a PSC are differentiated by EXP value on the label. The PSC of an L-LSP is explicitly signaled during LSP establishment. In L-LSP, PSC and EXP determine the QoS treatment. PSC determines the queue and EXP determines the WRED profile within that queue. Packet marking is done on EXP field. Using the MPLS Diff-Serv model, an MPLS service provider can offer differentiated services to customers sending IP traffic or MPLS traffic.

\section{B. DiffServ Aware Traffic Engineering}

Differentiated Services (DiffServ) enables scalable network designs with multiple classes of service. MPLS traffic engineering (TE) enables resource reservation, fault-tolerance, and optimization of transmission resources. MPLS DiffServ-TE combines the advantages of both DiffServ and TE. The result is the ability to give strict Quality of Service (QoS) guarantees while optimizing use of network resources.

MPLS TE operates at an aggregate level across all classes of service and as a result it cannot give bandwidth guarantees on a per class basis. The basic DiffServ aware TE requirement is to be able to make separate bandwidth reservations for different classes of traffic and give different forwarding behaviour based on the class [1]. This implies keeping track of how much bandwidth is available for each type of traffic at any given time on all routers throughout the network. For this purpose, the concept of a class type (CT) is introduced [10] as follows: The set of traffic trunks crossing a link, which is governed by a specific set of bandwidth constraints. CT is used for the purposes of link bandwidth allocation; constraint based routing, and admission control. A given traffic trunk belongs to the same CT at all links. The

IETF requires support of up to eight CTs referred to as CT0 through CT7. DiffServ-TE adds the available bandwidth for each of the eight CTs as a constraint that can be applied to a path. Therefore, CSPF is enhanced to take into account CT-specific bandwidth at a given priority as a constraint when computing a path. For the computation to succeed, the available bandwidth per-CT at all priority levels must be known for each link. One of the most important aspects of the available bandwidth calculation is the allocation of bandwidth among the different CTs. The percentage of the links bandwidth that a CT (or a group of CTs) may take up is called a bandwidth constraint (BC). There are two BC models: Maximum Allocation Model (MAM) and Russian Dolls Model (RDM) [1]. 


\section{CONCLUSION}

The aim of this paper was to clarify the QoS related terminology associated with MPLS networks. MPLS increases the QoS when used in extension with IP. The DiffServ architecture of MPLS and MPLS -TE are the features that make QoS better. The combined use of the MPLS DiffServ and MPLS TE is envisioned to provide end to end guaranteed QoS for multiservice traffic in IP networks.

\section{REFERENCES}

[1] Dongli Zhang and Dan Ionescu, "QoS performance analysis in deployment of DiffServ -aware MPLS traffic engineering", IEEE computer society, 2007, pp. 963-967.

[2] [2] Junaid Ahmed Zubairi. " Voice transport techniques over MPLS," IEEE, $p p$. 25-29.

[3] M. A. Rahman, A. H. Kabir, K. A. M. Lutfullah, M. Z. Hassan, and M. R. Amin, " Performance analysis and the study of the behavior of MPLS protocols", IEEE, 2008, pp. 226-229.

[4] Mahmoud M. Al-Quzwini and Sarmad K. Ibrahim, " Performance evaluation of traffic engineering signal protocols in IPv6 MPLS networks", Scientific Research, 2012, vol.4, pp. 298-305

[5] M. Kr. Porwal, A. Yadav and S. V. Charhate, "Traffic analysis of MPLS and Non-MPLS network including MPLS signaling protocols and traffic distribution in OSPF and MPLS", IEEE computer society, 2008 pp.187-192.

[6] O. Gure, B. K. Boyaci, and N. O. Unverdi,2010.Analysis of the service quality on MPLS networks, in Circuits and Systems for Communications (ECCSC), 2010 5th European Conference on, pp. 43-46.

[7] S. Rajagopalan1, E.R. Naganathan2, and P. Herbert Raj, "Ant Colony Optimization Based Congestion Control Algorithm for MPLS Network," IEEE, pp.214-223.

[8] 3] F. L. Faucheur and W. Lai, "Requirements for Support of DifferentiatedServices-aware MPLS Traffic Engineering," RFC 3564, July 2003.

[9] E. Rosen, A. Viswanathan, and R. Callon, "Multiprotocol Label Switching Architecture," RFC 3031 (Proposed Standard), Jan. 2001.

[10] F. L. Faucheur, L. Wu, B. Davie, S. Davari, P. Vaananen, R. Krishnan, P. Cheval, and J. Heinanen, "Multi-Protocol Label Switching (MPLS) Support of Differentiated Services," RFC 3270 (Proposed Standard), May 2002.

[11] Janusz Gozdecki, Andrzej Jajszczyk and Rafal Stankiewicz, "Quality of Service Terminology in IP networks", IEEE communication magazine, pp.153-159, March 2003.

[12] S. Blake, D. Black, M. Carlson, E. Davies, Z. Wang, and W. Weiss, “An Architecture for Differentiated Service," RFC 2475, Dec. 1998.

[13] K. I. Park, "QOS in packet networks", Vol. 779, Springer, 2005.

[14] W.C.Hardy, QoS Measurement and Evaluation ofTelecommunications Quality of Service, Wiley, 2001. 